\title{
Estados Unidos, la presidencia imperial: del poder regional al poder global
}

DOI: $10.32870 /$ mycp.v12i36.332

Abelardo Rodríguez Sumano ${ }^{1}$

$\mathrm{E}$ 1 objetivo de este artículo consiste en entender las fuerzas que marcaron el inicio de Estados Unidos como una presidencia imperial, los grupos de poder que gravitaron en torno a la expansión territorial y comercial que extendieron a la naciente potencia hacia el Atlántico y al Pacífico. ${ }^{2}$ La piedra de toque de esta transformación fue la guerra que la Casa Blanca y el Congreso declararon contra España en 1898 y que tenía como corolario ocupar Cuba, Puerto Rico y las Filipinas. A este impulso le siguió la adquisición de Hawai y la construcción del Canal de Panamá; situación que proyectó a Estados Unidos ${ }^{3}$ del hemisferio occidental a Europa y Asia, respectivamente. Asimismo, apuntó su rol como actor prominente en la escena mundial, que se desenvolvió a lo largo del siglo xx. Este crecimiento espectacular le permitió tejer, de igual forma, su "relación especial" con el Reino Unido y sentar las bases de una relación estratégica al inicio de un nuevo siglo.

\section{Sobre el imperio}

Antes de entrar directamente en el trabajo de análisis de este escrito, bien vale la pena detenernos por un instante en nuestra aproximación conceptual a la idea de imperio para brindar mayor consistencia al presente ensayo. Para

1. Profesor-investigador del Departamento de Estudios del Pacífico, Centro Universitario de Ciencias Sociales y Humanidades de la Universidad de Guadalajara. El autor agradece a las $C$. Belém Plasencia y Luisa Fernanda Cervantes por su valioso apoyo en este trabajo.

2. LaFeber, Walter, The New Empire: an Interpretation of American Expansion 1860-1898, Ithaca and Cornell University Press, Londres, 1963: vii.

3. Ferguson, Niall, Empire: how Britain made the modern world, Penguin Books, Londres, 2003: xxi. 
Stephen Peter Rosen, imperio es el ejercicio de las normas por una nación sobre otra con el objeto de regular su comportamiento interno y externo dentro de los Estados subordinados. ${ }^{4}$ En palabras de James O'Connor, es "la extensión del poder político por un Estado sobre otro". ${ }^{5}$

La tarea imperial consiste en la creación y manejo de un orden interestatal jerárquico, señala Peter Rosen. Un imperio debe también garantizar la seguridad y la estabilidad interna de sus partes constituyentes, extraer ingresos para pagar el costo del imperio, y asimilar las élites de sociedades no imperiales al corazón metropolitano, tareas que presuponen influencia sobre los asuntos internos de otras sociedades. ${ }^{6}$ Para Joseph Schumpeter, en el imperialismo "los ciudadanos y bienes de una nación pueden moverse en países extranjeros tan libremente como si esos países fueran políticamente propios". Todos estos elementos nos ayudan a entender el comportamiento de Estados Unidos antes y después de la guerra con España de 1898 y que dieran origen a la llamada presidencia imperial.

En este trabajo nos apoyamos en el concepto de Stephen Peter. Asimismo, cabe señalar que no es objeto del presente escrito apoyarnos en la explicación del imperio soviético de Lenin. ${ }^{7}$ Es importante apuntar también, que con el fin de la Segunda Guerra Mundial la literatura sobre el imperio quedó fuera del discurso y de las iniciativas que dieron forma al sistema internacional encabezado por la Organización de las Naciones Unidas. ${ }^{8}$ No obstante, una abundante literatura reemergió tras los ataques terroristas en Estados Unidos que desempolvaron nuevamente el concepto y le dieron vigencia por la presidencia de George W. Bush. ${ }^{9}$ Un punto de referencia central es la guerra con España en 1898; a la luz de ese interludio, creemos, se justifica el presente ensayo.

4. Rosen, Stephen Peter, "An Empire, If You Can Keep It". The National Interest. Spring, 2003: 51.

5. O'Connor, James, "The Meaning of Imperialism", en K. T. Fann, y Donald C. Hodges (eds.), Readings in U. S. Imperialism, Porter Sargent Publisher, Boston, 1971: 23.

6. Rosen, Peter, op. cit. p. 52.

7. Dougherty, James E., y Pfaltzgraff, Robert L. Jr., Teorías en pugna en las relaciones internacionales, Grupo Editor Latinoamericano, Buenos Aires, 1993.

8. Scott, Len, “International history 1945-1990", en Baylis, John, y Steve Smith, The Globalization of World Politics, An Introduction to International Relations, $2^{a}$ edición, University Press, 2001: 76.

9. Bender, Peter, America: The New Roman Empire?, Foreign Policy Research Institute, Winder, 2003; Griffin, Ray, The New Pearl Harbor, Disturbing Questions about the Bush Administration and 9/11, Olive Branch Press, Massachusetts, 2004. 
Estados Unidos, la presidencia imperial: del poder regional al poder global

\section{La fiebre expansionista}

El final del siglo XIX vaticinaba el cierre de un ciclo histórico en la historia de la presidencia de Estados Unidos. Seguros de sus logros expansionistas de la primera mitad del siglo con la anexión de Texas, Nuevo México, Arizona y California; ${ }^{10}$ firmes en la adquisición de Rusia, de más territorio de Alaska en 1867;11 fortalecidos tras la Guerra Civil entre el Norte y el Sur de la Unión Americana; revitalizados con la emergencia a gran escala de las grandes empresas, la industria ferroviaria,

La tarea imperial consiste en la creación y manejo de un orden interestatal jerárquico la invención del telégrafo y el teléfono que unieron y comunicaron a Estados Unidos de costa a costa y de norte a sur. ${ }^{12}$ La emergente potencia regional propició que ese gran boom atrajera inmigrantes de Polonia, Rusia, Italia, Grecia, Japón, China y México que, aunque eran en su mayoría pobres e iletrados, contribuyeron al gran empuje que Estados Unidos estaba construyendo en sus mocedades, vinculando a esa nación con Europa, Asia y América Latina. Esta transformación, sin precedentes en las Américas, también gestó un impresionante crecimiento poblacional, que entre 1870 y 1900 se disparó de 31 a 76 millones de habitantes. ${ }^{13}$

10. Henderson, Timothy J., A Glorious Defeat: Mexico and its War with the United States, Hill and Wang, Nueva York, 2007.

11. Para la compra de Alaska a Rusia véase http://www.library.state.ak.us/hist/cent/pca020html.

12. Históricamente la invención del teléfono se le ha atribuido al escocés-estadounidense Alexander Grahan Bell; no obstante, en junio de 2002 el Congreso de Estados Unidos reconoció que el teléfono fue concebido por un desconocido inmigrante italiano llamado Antonio Meucci. Increíble, ¿verdad? En la resolución, el Congreso reconoció que el teletrófono Meucci (así lo bautizó él) se mostró públicamente en Nueva York en 1860, 16 años antes de que Bell lo patentara. El veredicto estadounidense también asegura que "La vida y logros de Antonio Meucci deben ser reconocidos, así como su trabajo en la invención del teléfono". Entonces habrá que comenzar a cambiar los libros de historia (http://icarito.aconcagua1.copesa.cl/especiales/medios/ telefono.htm). Sin embargo, Estados Unidos lo industrializó en su escala global. Por su parte, el artista profesional Samuel Morse desarrolló y patentó de manera independiente el primer telégrafo de Estados Unidos. Para 1832 ya tenía diseñado un boceto de su telégrafo y estaba creando el sistema de alambres electromagnéticos para su funcionamiento.

A principios de enero de 1833, Morse realizó la primera demostración pública del telégrafo confeccionado por él. Dos años después apareció el primer modelo telegráfico desarrollado por Morse (http://www.mipunto.com/temas/2do_trimestre06/telegrafo.html).

13. Davidson, James West, y Gienapp, William E. et al., Nations of Nations: A Narrative History of the American Republic, McGraw-Hill, Nueva York, 1990: 686. 
Con esta energía electrizante (re)emergieron las tendencias imperialistas en el seno de la Unión Americana. Herederos de la Gran Bretaña, los estadounidenses crecieron y aprendieron a rivalizar con ella, y a partir de la segunda mitad del siglo XIX, a querer imitarlos y por supuesto, superarlos. Todavía existían remanentes coloniales del paso imperial de Londres, París, Madrid y Lisboa en el hemisferio occidental en la medida que nacían nuevos actores en el escenario mundial: Estados Unidos en las Américas, Alemania en Europa y Japón en Asia. Sin embargo, el motor de la "excepcionalidad" estadounidense se aglutina en la Revolución de Independencia (1776) y su forma de gobierno "libre y democrático", que fue por antonomasia antimonárquico. ${ }^{14}$ Esa "excepcionalidad" cuajó gracias al arraigo de la ideología de la divina providencia y el destino manifiesto apuntalado en sus instituciones, desarrollo económico y la defensa de la libre empresa. ${ }^{15}$ En este rubro existe una contradicción que vale la pena aclarar: una de las preocupaciones más apremiantes ${ }^{16}$ de Estados Unidos consistía en contener la influencia de Europa en este continente, para ser Washington el gran actor determinante en el devenir del hemisferio. En contraste, Estados Unidos no sólo copió y reverenció a Europa, sino que buscaba superarla, sin negar sus raíces en Occidente. ${ }^{17}$ No obstante, los estadounidenses se veían a sí mismos como los "verdaderos" propagadores del cristianismo protestante, la democracia representativa y el capitalismo transnacional. ${ }^{18}$

Ahora bien, el acelerado crecimiento de Estados Unidos amplió la interdependencia que estaba gestando esta nación con el resto del mundo a través del comercio, la industria de las armas y las comunicaciones, que se expresaron ideológica, política, militar y culturalmente. En efecto, fue un pequeño número de grupos de interés que en el corazón de sus entrañas transformaron la fisonomía de la política exterior estadounidense. Las asociaciones evangélicas, el lobby de la marina de guerra, el sector de negocios, los comerciantes agrícolas, y un selecto grupo de intelectuales y hombres de la élite política

14. Ferling, John, Almost a Miracle, The American Victory in the War of Independence, Oxford University Press, 2007: 135.

15. Ortega y Medina, Juan A., Destino manifiesto: sus razones históricas y su raíz teológica, Alianza Editorial Mexicana/Consejo Nacional para la Cultura y las Artes, México, 1989.

16. Para Estados Unidos, contener la influencia europea y consolidarse como el único actor de influencia del hemisferio.

17. Winter, Caroline, The Culture of Classicism: Ancient Greece and Roma in American Intellectual Life 1780-1910, The Johns Hopkins University Press, 2002: 9.

18. Gujahr, C. Paul, An American Bible, A History of the Good Book in the United States, 1777-1880, Stanford University Press, Stanford, California, 1999: 40. 
coadyuvaron en el surgimiento de una potencia imperial y transformaron el rol de Estados Unidos en el siglo Xx. ${ }^{19}$

La élite empresarial impulsó la carrera expansionista, que proponía nuevos mercados, abundantes recursos naturales y, por supuesto, mano de obra barata que trabajaba en Estados Unidos y al mismo tiempo se encontraba conectada a las cadenas productivas con representantes en Europa, Asia y América Latina. La construcción del ferrocarril y el desarrollo de puertos dinamizaron esta cadena comercial y financiera, que aumentó con la incorporación de comerciantes agrícolas e industriales a la fuerza laboral estadounidense.

Esta proyección fue conceptualizada e imaginada en un proyecto de gobierno por un selecto número de figuras simpatizantes con el imperialismo, entre quienes destacó Henry Brooks Adams, hijo del embajador Charles Francis Adam, nieto del presidente y secretario de Estado John Quincy Adams y bisnieto del presidente John Adams. A pesar de que Henry Brooks Adams no formaba parte de la clase política activa, su pensamiento era de gran importancia para sus amigos políticos como el senador Henry Cabot Lodge, el joven patriota Theodore Roosevelt y John Hay, amigo del ex presidente Abraham Lincoln, de quienes hablaremos más adelante.

Fue el fervor expansionista (impulso de nuevos mercados) e imperialista (apertura de nuevos mercados, más la adquisición y subordinación de nuevos territorios) revitalizado por los medios de comunicación afines a los intereses económicos, lo que distinguió a empresarios y políticos de la época en su alianza contra España en 1898. Ese proyecto buscó la construcción de un canal que uniera al Océano Atlántico con el Pacífico y controlara el Istmo de Tehuantepec, además de incorporar a la jurisdicción estadounidense las islas de Hawai y Cuba, respectivamente. El promotor de estas iniciativas era el senador por Massachusetts, Henry Cabot Lodge, quien además aseguraba que Estados Unidos estaba consagrado a edificar una civilización inalcanzable. ${ }^{20}$

En efecto, en el último cuarto del siglo XIX se consolidó la presencia regional de Estados Unidos en el hemisferio occidental. En 1893 el historiador Frederick Jackson Turner concibió una nueva teoría de la expansión, que sostenía: si las instituciones de Estados Unidos no se extienden más allá

19. Hendrickson, David C., "The Curious Case of American Hegemony: Imperial Aspirations and National Decline", World Policy Journal, vol. xxII, núm.2, verano, 2005: 3.

20. Irons, Peter, War Powers, How the Imperial Presidency Hijacked the Constitution, Metropolitan Books/Henry Holt and Company, Nueva York, 2005: 88 y 89. 
de sus límites fronterizos, se "resquebrajarán". ${ }^{21}$ Estos factores avivaron el ultranacionalismo. ${ }^{22}$ De esta forma se elevó al primer plano del poder en Washington el camino para la anexión de nuevos territorios, que se detuvo tras la guerra con México y la incorporación de más de la mitad de su territorio a la jurisdicción estadounidense.

En contrasentido, la profundización de la interdependencia convirtió a México en una nación complementaria respecto de Estados Unidos desde 1880. La prolongada estabilidad que proporcionaba la dictadura del general Porfirio Díaz (1877-1880 y 1884-1911) y las resistencias internas que se avivaron con la Guerra Civil calmaron los ánimos anexionistas al sur de su frontera al más alto nivel de la presidencia. ${ }^{23}$ Hacia el norte, Estados Unidos daba sus primeros grandes pasos hacia la integración comercial con Canadá y que se prolongarían a lo largo del siglo xx. ${ }^{24}$ En ambos casos los estadounidenses apostaron a que era más redituable profundizar el comercio y la inversión, que la anexión y la guerra con México y Canadá en el avance de su naciente proyecto global. (Sin embargo, ese curso hacia la integración regional de América del Norte que alcanzó su expresión más acabada con el TLCAN, fue replanteado profundamente tras el 11 de septiembre de 2001 y el nuevo perímetro de seguridad de América del Norte, y resurgieron los temores intervencionistas en México y Canadá de nueva cuenta como en el siglo XIX.)

En esa coyuntura histórica, otra variante del mismo proceso buscó extender su dominio al control de mares y puertos, expandir su industria y sector empresarial fuera de sus contornos territoriales. Lo anterior se vuelve más plausible si consideramos que en 1890 los ciudadanos estadounidenses ya no alcanzaban a consumir lo que producían. ${ }^{25}$ En 1893 la Unión Americana se convertía en la segunda fuerza en el comercio internacional después de

21. http://www.usspanishwar.org.

22. http://www.publichistory.org/evaluation/index.html (02/11/09).

23. No obstante, los intentos por adquirir más territorios de México no cesaron a lo largo de la segunda mitad del siglo xIx (Meyer, Lorenzo, y Vásquez Zoraida, Josefina, México frente a Estados Unidos: Un ensayo histórico 1776-1988, Fondo de Cultura Económico, México, 1989).

24. En 1867 Sir John A. MacDonald intentó reactivar el Tratado de Reciprocidad de 1854, un pequeño acuerdo de libre comercio que fue anulado por Estados Unidos en 1866. Éstos son los antecedentes del Tratado de Libre Comercio que entró en marcha entre Estados Unidos y Canadá el 1 de enero de 1989. Véase: Robert Maryse, Negotiating NAFTA, Explaining the outcome in culture, textiles, autos, and pharmaceuticals, University of Toronto Press, Toronto, 2000: 26.

25. Si no hubiera sido porque la anexión de Texas, Nuevo México, Arizona y California extendía el sistema de esclavitud de norte a sur y hacia el oeste, quizá Estados Unidos no hubiera tenido la guerra civil, y entonces la carrera expansionista hubiera continuado. Pero la esclavitud era 
Estados Unidos, la presidencia imperial: del poder regional al poder global

Gran Bretaña y lideraba la exportación de petróleo y algodón. Asimismo, la marina estadounidense se convirtió en la número siete en la escala mundial y la tercera 10 años más tarde. En ese mismo año (1893) Theodore Roosevelt convocaba a la clase política para que Washington interviniera en el conflicto entre España y Cuba, disputa que se remontaba a 1868 cuando La Habana inició su lucha de independencia del imperio ibérico. ${ }^{26}$ Roosevelt era un ferviente admirador de la capacidad de fuerza en ultramar de los británicos, que dominaron el escenario mundial del siglo XVIII y finales del xIX. En la lectura estadounidense de la época, el Caribe vislumbró una oportunidad histórica para ensayar la travesía de la marina de guerra en la región.

En una carta fechada el 5 de abril de 1896, Roosevelt dejaba testimonio de algunas de sus motivaciones respecto del papel de Estados Unidos en la escena internacional, al escribir a su cuñado, el Almirante William Cowles:

A pesar de que me siento sólido en las cuestiones de la reforma municipal o la reforma al servicio civil, me siento aún más sólido sobre la cuestión de nuestra actitud hacia el mundo exterior, lo cual implica de la defensa de la costa a una marina de primera clase, al desarrollo vigoroso de una política exterior [...] creo que sería adecuado transformar nuestra política con la remoción de todos los poderes europeos de sus colonias que ellos poseen en el hemisferio occidental. ${ }^{27}$

Para hacer viable está visión, Estados Unidos debía todavía negociar un entendimiento clave con la Gran Bretaña sobre el hemisferio occidental y sobre su papel global; y dos, remover a España de este continente, que pasaba por una transformación sustancial y a fondo de la política exterior de la Casa Blanca en el hemisferio, como veremos más adelante.

\section{Cuba en la mira}

El interés de Estados Unidos por Cuba — apunta el historiador Louis Pérezes mucho más añejo y profundo: éste se remonta a la presidencia de Thomas Jefferson (1800-1809) como un deseo inevitable que llevaría en algún punto

parte misma del modelo que Estados Unidos construyó desde la fundación de su Estado nacional. Por lo tanto, el expansionismo cobró un rostro distinto: la creación de protectorados.

26. Ferrer, Ada, Insurgent Cuba, intro.

27. Citado en Mathew Crenson y Benjamin Ginsberg, Presidential Power: Unchecked and Unbalanced, Norton, Nueva York, 2007: 114. 
de su historia a la anexión. ${ }^{28}$ Ciertamente, los estadounidenses desde 1803 (la compra de la Louisiana a Francia) empezaron a desplazar a los europeos que poseían territorio en el continente americano, propiciaron con absoluta decisión la independencia de América Latina y derrotaron a Londres en 1814; cuestión que los reivindicó como potencia regional de las Américas, antes que ninguna otra colonia novohispana alcanzara la independencia de España. La tarea de la supremacía de Estados Unidos sobre Europa en lo general y sobre España en particular, pasó por la compra de la Florida a España en 1819, la consumación de la independencia de México en 1821, la definición de la Doctrina Monroe en 1823, la anexión de Texas a Estados Unidos en 1845, la guerra con México y la adquisición de Nuevo México, Arizona y California en 1848, así como la compra de Alaska a Rusia en 1867.

Dicho de otra manera, Estados Unidos le tenía medido el pulso a España por alrededor de un siglo. La compra de la Florida a Madrid en 1819 y la definición de la Doctrina Monroe en $1823^{29}$ reorientaron el pensamiento de Jefferson, que se acabó de pulir con el surgimiento de El destino manifiesto en $1839^{30}$ sobre la extensión de las ideas y las instituciones estadounidenses más allá de sus límites fronterizos durante el siglo XIX. No obstante, esto no significó el fin de las relaciones socioculturales entre Cuba y el sur de la Florida. Como tampoco se cortó de tajo con la vinculación sociocultural entre California, Texas, Nuevo México y Arizona una vez que estos territorios dejaron de pertenecer a México. Por el contrario, estas relaciones ayudaron a establecer vínculos binacionales económica, cultural y políticamente - como lo hicieron con los estados de la Unión en la Costa del Pacífico y Asia. ${ }^{31}$

28. Pérez, Louis A. Jr., The War of 1898: The United States and Cuba in History and Historiography, The University of North Carolina Press, Chapel Hill y Londres, 1998: 42.

29. La Doctrina Monroe fue una política exterior de Estados Unidos expresada por primera vez el 2 de diciembre de 1823 por el presidente James Monroe en su séptimo mensaje anual al Congreso estadounidense. En éste expresaba que cualquier intento de cualquier nación europea de colonizar o interferir en el continente americano sería percibido como un acto de agresión, por lo que Estados Unidos se vería forzado a intervenir. Expresaba además que Estados Unidos no intervendría en las colonias europeas existentes ni en los asuntos internos de las naciones europeas (http://avalon.law.yale.edu/19th_century/monroe.asp).

30. El destino manifiesto es un documento escrito en 1839 por John O'Sullyvan, en el cual se ensalza el nacimiento de la nación Americana y su "perfecta unión", el destino de América, además de invocar el patriotismo americano y la grandiosidad de la "era americana" (http:// www.civicsonline.org/library/formatted/texts/manifest_destiny.html).

31. "La política exterior estadounidense a finales del siglo xIx y comienzos del siglo xx: 18701914", Gobierno de Castilla y León (www.artehistoria.jcyl.es/historia/contextos/2754.htm). 
Empero, el periodo en el que se hilvana una relación más estrecha entre Cuba y Estados Unidos está ligada al resultado de la Guerra de los Diez Años (1868-1878), a la Pequeña Guerra de 1879 y 1880 y, desde luego, a la preparación de la Guerra de Independencia de 1895 a 1898 de España. A lo largo de esos casi 30 años el conflicto fue acompañado por el creciente comercio y la inversión de Washington en La Habana, que coincidió con la Revolución Industrial que Estados Unidos impulsó tras la Guerra Civil. Durante la última década del siglo xix la inversión de Estados Unidos en Cuba aumentó a los 50 millones de dólares y su intercambio comercial sobrepasó los 100 millones de dólares anualmente. ${ }^{32}$ Una de las iniciativas más importantes que se gestaron entre España y Estados Unidos fue el Tratado de Reciprocidad, que incrementó el intercambio comercial entre la Florida y Cuba. Documento que inauguró la inmigración masiva de trabajadores cubanos en Key West, Tampa y se prolongó por toda la Florida y otros estados de la Unión Americana. Mientras tanto, el proceso de liberación de Cuba de España ayudó a construir su identidad nacional y su nacionalismo, y que se enfrentaría al concluir el siglo con Estados Unidos. ${ }^{33}$

\section{Grover Cleveland, un represor moderado}

En 1893 Grover Cleveland alcanzó la Casa Blanca, fecha que coincidió con una importante depresión económica que lo llevó a la represión de los trabajadores liderados por el jefe sindical Jacob Coxey. Cleveland llegaba por segunda ocasión no consecutiva, después de haber ocupado la presidencia entre 1885 y 1889, en medio de una fuerte depresión económica. De igual forma, la prioridad del demócrata se concentró en el plano interno y evitó la confrontación externa a toda costa. Sin embargo, no pudo controlar ambas cámaras del Congreso en su arribo.

En este sentido, los halcones de la guerra recuperaron la mayoría en el poder legislativo en 1894 (al igual que 100 años después, cuando los republicanos controlaron la mayoría en ambas cámaras durante el primer periodo presidencial de Bill Clinton) como preludio de su ascenso en 1897 con William

32. World Powers, op. cit., p. 89.

33. Éste es un punto de referencia muy importante que coadyuvó en la Revolución Cubana de 1958 y permitió enfrentar a Estados Unidos y concretar la independencia que fue frustrada por Washington en 1898. Para la Revolución Cubana, 1898 fue un aliciente muy profundo que impulsó el nacionalismo antiestadounidense. 
McKinley a la cabeza. Los congresistas hacían eco de los grandes empresarios y de la emergencia espectacular de los medios de comunicación, que presionaron a Cleveland para intervenir en La Habana.

No obstante, el presidente demócrata se distinguió en materia de política exterior por impulsar una visión más moderada sobre el uso de la fuerza respecto de Cuba. Es importante dejar constancia en este texto de que al igual que durante la guerra contra México, en la guerra contra Cuba existían voces disidentes dentro de Estados Unidos que se oponían a la cruzada militar, tanto en la Iglesia católica como en el movimiento laboral y en la comunidad afroamericana tras la difusión de los últimos tres años de la guerra de Cuba contra España en la prensa de aquel país. Sin embargo, en 1896 el Congreso promovía una "resolución amigable" entre La Habana y Madrid, pero ésta era negada en ultramar por el gobierno español. Consecuentemente, los representantes de Capitol Hill apuntaban hacia una salida militar contra España para que Cuba alcanzara su "libertad e independencia." En forma contraria, Cleveland sostuvo "que no movilizaría a la marina de guerra para la confrontación bélica” mientras él fuera presidente. ${ }^{34}$

\section{William McKinley y los tambores de la guerra}

Al año siguiente, en 1897, las coordenadas políticas se modificaban sustancialmente. A la Casa Blanca arribó un miembro del Partido Republicano, miembro de la élite empresarial, veterano de la Guerra Civil, y diputado en la Cámara de Representantes que promulgó la defensa de los hombres de negocios ante la competencia extranjera. ${ }^{35}$ William McKinley, de Niles, Ohio (1843), compitió en 1886 contra el demócrata William Jennings Bryan, con el impulso toral del sector empresarial y el sector conservador. Su victoria no es entendible sin el apoyo de los empresarios, quienes veían con desdén a los correligionarios, obreros y agricultores seguidores de Jennings Bryan.

La victoria de McKinley ya tenía una ansiosa agenda: la intervención en Cuba. En el inicio la Casa Blanca buscó una salida diplomática. ${ }^{36}$ Empero, la mentalidad de la guerra ya estaba en camino y los argumentos se hacían más

34. Freddy, Mark, y Tood Davis, The New Big Book of U. S. Presidents, Courage Books/Running Press Books Publishers, Filadelfia, 2000: 55.

35. Freddy, Mark, op. cit, p. 57.

36. Montojo Pan, Juan, “¿Qué habría ocurrido si España hubiese evitado la Guerra con Estados Unidos en 1898”, en Townson, Nigel, Historia virtual de España (1870-2004), Taurus, 2004: 64. 
sustantivos. En este tenor figuró la silueta de Valeriano Wayler, el general español cuyo sistema había provocado gran inconformidad en Estados Unidos; él era responsable de la muerte de unos 150,000 cubanos, que según los estadounidenses habían muerto en "condiciones terribles". Ramón Blanco, su sucesor, intentaría moderar la "carnicería" que el presidente Antonio Cánovas del Castillo había permitido, quien fue asesinado el 8 de agosto de 1897. Su sucesor, Praxes Mateo Sagasti, junto con su ministro de Ultramar, Segismundo Moret, promovieron la autonomía de Cuba y Puerto Rico el 27 de noviembre de 1897, demanda explicita de Cleveland. Esta política buscaba flexibilizar la colonia y abrir un nuevo cambio comercial entre Cuba y Estados Unidos. ${ }^{37}$

Sin embargo, con la llegada de McKinley al poder, la aparente moderación de España ya no tenía peso en el decurso de las cosas respecto de la eventual guerra entre ambas naciones. "Washington estaba horrorizado y conmovido" por la pobreza y la indigencia en la que habían caído los cubanos. ${ }^{38} \mathrm{~A}$ principios de 1898 España ya había acercado 200,000 hombres en La Habana y su secretario de Relaciones Exteriores promovía una liga de potencias europeas para neutralizar la participación de Estados Unidos en el eventual conflicto. De entrada, la Gran Bretaña la rechazó, fueron desdeñados por Rusia, Alemania, Francia y el imperio Austro-húngaro simpatizó con la monarquía ibérica, pero nada más. Por si fuera poca cosa, la aparición de una misiva personal del embajador español en Washington, Enrique Dupuy de Lome, en la que denostaba al presidente republicano, se eclipsaba con el hundimiento "accidental" del buque Maine en las aguas de La Habana. ${ }^{39}$ La prensa sensacionalista, encabezada por el Journal de Nueva Cork, atizaron las intrigas de la confrontación entre Washington y Madrid.

Posteriormente el embajador estadounidense en España, Stewart Woodford, entregó lo que vendría a ser un ultimátum de Estados Unidos al gobierno español para deponer el fuego unilateralmente, teniendo como árbitro al presidente McKinley. El gobierno español decidió entonces enfrentar la guerra con Estados Unidos que sufrir la "humillación", a sabiendas de que la posibilidad de la victoria era muy remota en una guerra contra ese país. Pero lo que estaba en juego era incluso algo más que sus colonias: la monarquía

37. Montojo Pan, op. cit., p. 65.

38. Nevins, Allan, y Henry Steele, Breve historia de los Estados Unidos, Fondo de Cultura Económica, 1994: 359 y 360.

39. Montojo Pan, op. cit, p. 66. 
y el sistema parlamentario. Y en el fondo de un imperio de 400 años - ya agonizante- estaba en juego: la defensa del "honor nacional." ¿De qué le sirvieron su moderación inicial y su posterior soberbia parcial?

Para Estados Unidos el hundimiento del Maine lo justificaba todo. ${ }^{40}$ Según los españoles el hundimiento había sido "incidental", mientras que para los estadounidenses fue una "provocación" inadmisible. (No obstante, cabe agregar que en pleno siglo XXI no existen pruebas plenamente convincentes de lo ocurrido con el Maine hace más de un siglo.) Una comisión investigadora de la época sólo atinó a "revelar" que la explosión se produjo al interior del buque. Los especialistas sobre el tema no se detienen en deshilvanar las causas de la conspiración, sino en las consecuencias de los hechos: tocar los tambores de la guerra. ${ }^{41}$ Sin embargo, ¿qué pasó realmente con el hundimiento del Maine? Queda pendiente una investigación de mayor alcance sobre el contexto y las causas de la guerra entre Estados Unidos y España, que futuras investigaciones deberán desentrañar.

En todo caso, para Estados Unidos la ocasión era "perfecta”, y la guerra, popular. El 11 de abril el presidente enviaba al Congreso una declaratoria bélica, una acción aplaudida y, sin embargo, innecesaria. El 20 de abril de 1898 ambas cámaras del Congreso inscribían una resolución promovida por el senador republicano, Henry Teller, de Colorado, en la que llamaban a la "liberación e independencia de Cuba". La lucha daba comienzo el 1 de mayo del mismo año y en 10 semanas los estadounidenses habían ya despachado a los peninsulares. Se trataba del conflicto que más ganancias le habían producido, y la más rápida que había enfrentado la Unión Americana hasta entonces.

\section{La guerra contra España y sus consecuencias}

En sintonía con el espíritu de la época, el presidente autorizó el uso de la marina de guerra para "preservar la soberanía de La Habana". Autorización que fue ratificada oficialmente por el Congreso el 25 de abril de 1898 con 311 votos a favor y seis en contra en la Cámara de Representantes, y con 42 votos a favor y 35 en contra en el Senado.

40. Millet, Allan, y Maslowsi, Meter, For the Common Defense: A Military History of The United States of America, The Free Press, 1994: 284.

41. Millet y Maslowsi, op. cit., p. 287. 
Una vez que la guerra se hizo efectiva, el presidente, como comandante en jefe instaló en la Casa Blanca el war room que reorganizó una infraestructura que lo mantuviera comunicado telefónicamente con su gabinete y el Congreso, además de maximizar el uso del telégrafo. La estrategia consistía en propinar golpes pequeños pero demoledores al enemigo, que en cascada dieran una sensación de erosión de su liderazgo con el fin de quebrar su estructura, que además se encontraba lejos de la metrópoli. Asimismo, el presidente se convirtió en el puente entre el ejército y la marina; situación que permitió una cooperación maestra entre ambos sectores para el intercambio de comunicación y preparación de la estrategia militar, a pesar de las tensiones entre ambas entidades de la defensa estadounidense.

España se encontraba mal organizada y distante para enfrentar la guerra. Mientras tanto, el Congreso de Estados Unidos aprobó un respaldo de 50 millones de dólares para la acción bélica, fondos que en su mayoría se fueron a la marina. Asimismo, la planeación estratégica de la Casa Blanca consideraba atacar a España en su territorio por medio de buques que patrullaban el Atlántico, iniciativa que resultó innecesaria por la incapacidad de las fuerzas españolas en el Caribe. Pero Washington pudo movilizar escuadrones desde Tampa y Key West en la Florida de una forma magistral que le daban una ventaja vertiginosa sobre las tropas españolas, que además rodeaban las costas de Cuba y entraban en San Juan, Puerto Rico, con relativa facilidad y atacaban en Manila con prontitud y eficacia. Los batallones se nutrían de las reservas de San Francisco, Nueva Orleáns, Key West, Mobile y Camp Alger.

En este marco el Congreso permaneció atento y otorgó mayor presupuesto, que sirvió para mejorar la infraestructura en el plano interno en el corredor del sur de Estados Unidos, que movilizó recursos hacia el sur de la Florida y los impulsó. La guerra fungió también como un elemento a favor de la unidad nacional, que se había erosionado durante la Guerra Civil. En suma, la maquinaria bélica coronada por el presidente de la república ponía en marcha la renovación de su flota, la relación estratégica que se operaba desde el Congreso, el proyecto económico del Departamento del Tesoro y el trabajo de la Casa Blanca con el resto de la Unión para movilizar tropas, infraestructura y recursos. El apoyo de la prensa fue clave para reivindicar el patriotismo.

Una vez que España aceptó la derrota, el Departamento de Guerra ejecutó la invasión de Puerto Rico, que desde entonces a la fecha perdió su soberanía y se convirtió en protectorado de Estados Unidos. Antes de continuar con este trabajo es pertinente formular algunas preguntas: ¿cuál era la relación entre 
la "liberación e independencia de Cuba" con la incursión militar de Estados Unidos en Puerto Rico y Filipinas? Y ¿cuál era la relación entre la Doctrina Monroe y las Filipinas?

\section{Surgimiento de la presidencia imperial}

Theodore Roosevelt emergió como un líder natural del conflicto con Cuba tras renunciar a su posición de subsecretario en la marina y enfilarse como voluntario en la isla caribeña. A su regreso a la Unión Americana en 1898 se convirtió en celebridad, lo que lo llevó a la gubernatura de Nueva Cork, y al final del último año del primer gobierno de MacKinley fue nombrado secretario de Estado. "Su gloria se alcanzó a expensas de un débil enemigo, pues la resistencia ofrecida por él fue digna de lástima [...]" asegura el historiador Allan Nevis. Y continúa: "Nuestras victorias se podrían atribuir en parte a la audacia y el valor, pero en parte aún más a la debilidad española. Y como trasfondo de estas victorias existió un historial de corrupción, ineficiencia y torpeza burocrática." ${ }^{42}$ En efecto, la guerra contra España había dejado al descubierto las carencias de la flota estadounidense, que debían arreglarse y, en todo caso, superarse. Tarea que revolucionaría el papel de sus tropas, que pasaron por la invasión del puerto de Veracruz en México en 1914, pero de manera total en la Primera Guerra Mundial, a la que ingresó Estados Unidos entre 1917 y 1918.

Para la reelección del hombre de Ohio, invitó como compañero de fórmula a la vicepresidencia al "héroe" Teddy Roosevelt. Hasta antes de la guerra con España Estados Unidos no poseía colonias; después acuñó claramente el título de imperio guiado por la presidencia; sí, una presidencia imperial que sustituía al imperio español en el hemisferio occidental. La victoria de MacKinley demostraba que la guerra había sido muy popular en la medida que Estados Unidos transformaba su nomenclatura de una democracia republicana a una democracia imperial, la cual se ejercitó desde el arrebato de Nuevo México, Arizona y California en 1848 a México.

Si el objetivo final del Congreso y la presidencia hubiera sido la independencia de Cuba, no hubiera existido una violación a la Constitución de Estados Unidos, asegura Meter Irons en su War Powers; por ello se convirtió

42. Nevins y Morris, op. cit., pp. 362 y 363. 
en imperio ${ }^{43}$ Más aún, Estados Unidos entró en una contradicción fenomenal debido a que su lucha de independencia y la edificación de su gobierno buscaban romper con la monarquía británica, y ahora por el peso de los hechos se convertían en una democracia hegemónica que subordinó a las naciones conquistadas y extrajo la esencia de su soberanía; precisamente a lo que se oponían los antifederalistas en 1789 con la inauguración de la presidencia en Estados Unidos. ${ }^{44}$

Tras el botín que significó Cuba, La Habana tuvo que adoptar una Constitución ad hoc a los intereses de la Casa Blanca a través de la Enmienda Platt. ${ }^{45}$ Una Constitución que le obligaba a vender algodón, azúcar y estacionar bases militares provenientes de Estados Unidos, medida que le abrió la puerta a Washington para ocupar Guantánamo en forma permanente y ocasionalmente estacionar bases militares. ${ }^{46}$ Irons sostiene que esta trayectoria se repitió tras la invasión de Estados Unidos a Japón y Alemania, al finalizar la Segunda Guerra Mundial, y al Irak de Saddam Hussein en 2003. En todos esos casos los países ocupados elaboran Constituciones ad hoc a los intereses de Estados Unidos, pero esa discusión deberá ser parte de otro trabajo.

Bajo estas condiciones de fuerza, España cedió además a Estados Unidos las Filipinas por medio del Tratado de Paz de 1899, junto con Puerto Rico y la isla del Pacífico de Guam por un pago de 20 millones de dólares. En estas circunstancias, Estados Unidos entraba al siglo xx como un imperio; éste tuvo que enfrentar por tres años resistencias prolongadas de las Filipinas, que llevaban años peleando contra España por su liberación. No obstante, a los

43. War Powers, op. cit., p. 91.

44. Milkis, Sydeney M., y Nelson, Michael, The American Presidency: Origins and Development 1776-2002, CQ Press, Washington, DC, 2003: 67.

45. Enmienda Platt 1901: nombrada así gracias al senador de Connecticut Orville Platt. Básicamente la enmienda, que está dividida en ocho artículos, prohíbe a Cuba firmar cualquier tratado con cualquier poder extranjero que atente contra la independencia de Cuba o tenga motivos militares, que el gobierno de Cuba no contraiga ninguna deuda con ningún país; Estados Unidos tiene el derecho de intervenir en la isla para preservar su independencia y para mantener un gobierno adecuado para la protección de la vida y la libertad individual de los cubanos; que el gobierno cubano se comprometa a implementar y desarrollar planes para el saneamiento de la isla, para prevenir enfermedades y asegurar a su gente, su comercio y los habitantes estadounidenses que ahí residan, y finalmente que con la finalidad de que EUA pueda mantener la independencia de Cuba y proteger a sus habitantes; el gobierno cubano deberá ceder o vender a EUA las tierras necesarias para poder ser utilizadas como estaciones navales (http://www.mtholyoke.edu/acad/intrel/platt.htm http://www.state.gov/r/pa/ho/ time/ip/86557.htm). Revisado el de 19 enero de 2010:10.

46. Irons, op. cit., p. 92. 
grandes empresarios estadounidenses les encantaba por la posición "excepcional" de Manila en el Pacífico; oportunidad para "apoderarse" del extenso comercio chino.$^{47}$ Esta situación le valió a Washington para implementar el Protocolo Bóxer de $1901^{48}$ a China junto con Gran Bretaña, Francia, Rusia, Alemania y Japón con el afán de proteger "los intereses comerciales y vidas" de Estados Unidos en el exterior.

\section{Redefinición de la "relación especial" con Gran Bretaña y otras naciones}

En todo caso, la importancia estratégica de Filipinas residía en que su posición geográfica otorgaba a Estados Unidos una ventana no solamente hacia China, sino a Japón, Indochina y al amplio mundo asiático. La guerra contra España le indicaba a Washington que era necesario ajustar su relación con Gran Bretaña para redefinir el papel de Europa en las Americas. Fue a raíz del viaje del buque Oregon, en el que tuvieron que pasar de la costa del Pacífico, rodeando el Cabo de Hornos hasta aguas cubanas, que se justificó la necesidad de construir un canal en el Istmo de Tehuantepec que desembocaría en un entendimiento compartido para transitar a través del Istmo entre Estados Unidos y Gran Bretaña, y así unir el Atlántico con el Pacífico.

Con esto se establecieron las bases históricas de la relación de Estados Unidos hacia sus colonias. Puerto Rico jamás recuperó su soberanía; Cuba —que estuvo ocupada por España desde 1511 hasta 1898, y a partir de ese año por Estados Unidos- alcanzó por fin su independencia en 1958 a través de la Revolución. Las Filipinas fueron ocupadas y alcanzaron su independencia hasta el fin de la Segunda Guerra Mundial. Después Estados Unidos se apropió de Panamá, lo que le dio comunicación al Océano Atlántico con el Pacífico, y que le llevó a Hawai por su inercia imperial. Para esto, los estadounidenses se encontraban en esa isla con una situación predominante desde 1887 y forti-

47. Nations of Nations, op. cit., p. 817.

48. El Protocolo Boxer fue firmado el 7 de septiembre de 1901 entre el emperador Qing de China y la Alianza de los Ocho Países (Austria-Hungría, Francia, Alemania, Italia, Japón, Rusia, Reino Unido y Estados Unidos) después de la derrota de China en la intervención para deponer a la rebelión Boxer en manos de la Fuerza Expedicionaria de los Ocho Poderes. Está compuesto por 12 artículos, entre los que se destaca el pago de una indemnización de 480 millones de taels de plata a las ocho naciones involucradas. El emperador debía permitir la instalación de tropas extranjeras en Pekín y la prohibición de importar armas y municiones durante dos años (http://www.international.ucla.edu/asia/article.asp?parentid=18133). 
ficaron su posición en Pearl Harbor. Estas transformaciones modificaron los límites territoriales de Estados Unidos al inicio del siglo xx y le permitieron desarrollar las bases de la relación especial con Gran Bretaña, que redefinió de igual forma las bases del sistema internacional en la primera mitad del siglo $\mathrm{xX}$, que pasan por las dos guerras mundiales subsiguientes.

En 1899 se efectuó, con un importante número de países, lo que fue la primera reunión de naciones sudamericanas en Washington, conocida como la Conferencia Panamericana. Tras la Guerra Civil y en medio del impresionante crecimiento económico de Estados Unidos y su subsiguiente expansionismo, la nueva nación, junto al imperio británico pudo tejer cabo a cabo la "relación especial" entre Washington y Londres respecto de su papel en el mundo en relación con disputas comerciales que se resolvieron con arbitraje, coadyuvando al tejido de su relación especial. Por ejemplo, ambas naciones pudieron institucionalizar un arbitraje internacional en Ginebra tras los choques del Alabama y las reclamaciones estadounidenses a los británicos durante la Guerra Civil (1861-1865).

El veredicto del arbitraje se inclinó a favor de Estados Unidos, donde Gran Bretaña debía pagar 15,500 millones de dólares; los británicos pagaron y se solucionó el problema. En una disputa con Canadá sobre las demarcaciones entre las aguas del norte para la pesca de focas, una vez más el arbitraje internacional deliberó, pero esta vez a favor de los británicos. En 1895 Estados Unidos y Gran Bretaña estuvieron al borde de la guerra una vez más por los límites fronterizos entre la Guayana Británica y Venezuela; el presidente Cleveland amenazó con la guerra en el Congreso; empero, los británicos aceptaron la jurisdicción de la Doctrina Monroe impulsada por Washington, que en un principio buscaban desatender. Sin embargo, británicos y estadounidenses sostuvieron que los intereses de ambas naciones eran más grandes que los límites territoriales entre la Guayana y Venezuela. Ambas potencias profundizaban así su admiración, respeto mutuo y futura cooperación.

En medio de esta tremenda transformación cayó asesinado el presidente MacKinley el 14 de septiembre de 1901. En el puesto tutelar le sucedió el héroe de guerra Theodore Roosevelt, quien profundizó el rostro de la política exterior estadounidense al iniciar el siglo XX.

El flamante presidente impulsó la política de las puertas abiertas al comercio internacional en China, que tenía un gran futuro para el mercado mundial, política que se remonta a 1840. Por otra parte, Estados Unidos se inclinó hacia América Latina de una forma mucho más tutelar. Y por la otra, Roosevelt 
exploró su nueva posición de gobernante de una potencia mundial al buscar una salida diplomática con el fin de concluir la guerra entre Rusia y Japón en 1905, así como el apoyo del gobierno republicano a las añejas disputas entre Alemania y Francia respecto de posiciones en el Norte de África.

Lo realmente importante de Estados Unidos en esa época fue su papel en Filipinas, el Caribe y en Panamá, y por supuesto la relación con Gran Bretaña. De esta forma, ambas naciones consolidaron su relación con un tenue entendimiento sobre el Lejano Oriente, el Caribe y el Atlántico. Con la construcción del Canal de Panamá, Gran Bretaña renunció a sus privilegios sobre el Istmo, lo que se remonta al Tratado Clayton-Bulwer de 1850. Asimismo, Gran Bretaña ayudó a establecer los límites entre Estados Unidos y Canadá a favor de Washington en la frontera con Alaska. Por si lo anterior fuera poco, en 1906 Gran Bretaña retiró su flotilla de Bermudas que vigilaban las Antillas, agraciando a los estadounidenses.

Para que la construcción del Canal se hiciera efectiva, Estados Unidos promovió la independencia de Panamá ${ }^{49}$ y de esa forma estableció una posición que le permitiera subordinar a esa nación. Con esta estrategia la nueva república echó a volar la construcción del Canal y avivó la inconformidad latinoamericana. Pero a Roosevelt le importaban muy poco las protestas al sur del Río Bravo. Sin embargo, ese entendimiento estratégico de principios del siglo xx les permitió a Londres y a Washington cultivar y profundizar el entendimiento anglo-estadounidense en las Américas y de esa manera apuntar acuerdos y estrategias futuras que habrían de ponerse a prueba en las dos guerras mundiales subsiguientes. ${ }^{50}$

De esa forma se ajustó la Doctrina Monroe tras la tercera conferencia panamericana, en la que Washington aseguró que Estados Unidos tutelaría la viabilidad de las "pobres" y "mal gobernadas" naciones latinoamericanas, lo que concluyó en una serie de protectorados e intervenciones militares en el hemisferio que se modificaron, dependiendo del gobierno bajo la presidencia

49. Fue en 1903 cuando Estados Unidos firmó un tratado con Colombia para construir un canal en el Istmo de Panamá, que en ese entonces era un departamento del gobierno colombiano; el tratado era Herrán-Hay, que no fue ratificado por el gobierno de Colombia; esto fue un incentivo o un pretexto para que Estados Unidos apoyara las ambiciones separatistas panameñas (http://www.critica.com.pa/archivo/historia/1903.html).

50. White, Brian, "British Foreign Policy: Continuity and Transformation", en Ryan K. Beasley, et al. (eds.), Foreign Policy in Domestic Comparative Perspective and International Influences on State Behavior, CQ Press, A division of Congressional Quarterly, 2002: 24. 
de su sucesor, el secretario de Guerra William Taft, quien diseñó y ejecutó la diplomacia del dólar. Sin embargo, la Doctrina Monroe no fue aceptada por el grueso de los países latinoamericanos y México la cuestionó con energía.

El protectorado incluía estacionar bases militares y retirarlas cuando Washington lo considerara necesario. En 1906, 1912, 1917 y 1920 las tropas estadounidenses desembarcaron en Cuba, no para garantizar su "independencia y libertad" sino para reprimir a los rebeldes que se oponían a la ocupación estadounidense. El ejercicio de lo que sucedió en Cuba fue una formalidad constitucional al declarar la guerra, pero fueron los intereses políticos y económicos los que determinaron el papel de Estados Unidos en el ocaso del siglo XIX y en el amanecer del aún impredecible siglo XX.

\section{Conclusión}

Un nuevo debate sobre el imperio - aunque sin consenso- surgió dentro y fuera de la Unión Americana a raíz de lo que se conoció como la Doctrina Bush, que consistió en ajustar el argumento de la supremacía militar a nivel global y que alcanzó su cúspide en la Doctrina de Seguridad Nacional de 2002, la cual apuntaba la posibilidad de atacar a otra nación, si así lo estimaba necesario para sus intereses estratégicos de largo plazo. Esa estrategia desempolvó el estudio - cambio y continuidad, similitudes y diferencias - de las diversas guerras de Estados Unidos a lo largo de su historia y se remontan a la guerra de independencia de 1776, la guerra contra Inglaterra en 1812, la guerra contra México en 1846-47, la guerra contra España en 1898 y la participación de Estados Unidos en dos guerras mundiales.

Asimismo, permitió ventilar al interior de la propia administración Bush, ideas imperiales que se creían superadas, las cuales son consistentes con un interludio amplio de supremacía militar y política de Estados Unidos a lo largo de su historia y que se remontan con toda claridad a 1898. De igual forma, permiten identificar una constante en el avance de Estados Unidos en el sistema internacional: la súper potencia se había extendido territorialmente, no sólo había delineado una presencia militar incuestionable sino que había adquirido mayor territorio y le había permitido trazar objetivos cada vez de mayor alcance e influencia global, así como el hecho de precisar su hegemonía hemisférica desde el inicio del siglo xx.

Tras los ataques terroristas del 11 de septiembre de 2001, Estados Unidos impulsó una guerra contra Afganistán y posteriormente contra Irak, y 
había rediseñado el tablero de sus intereses estratégicos a todo el mundo. Al igual que tras el surgimiento de la presidencia imperial, la Casa Blanca de Bush ensanchó las capacidades institucionales y de radio de acción territorial de la presidencia estadounidense en el siglo xxi. Sin embargo, la capacidad de acción de la Unión Americana para hacer valer sus intereses se encontró con severos estragos de planeación en contra de sus "enemigos" en el siglo XXI.

Recapitulando, en la guerra contra España, Washington procreó una presidencia imperial que se encontraría con otras potencias con similares ambiciones, aunque con distintos objetivos, como Italia, Alemania y Japón en la Segunda Guerra Mundial. No obstante, con el fin de la Segunda Guerra Mundial la retórica imperial salió de la estructura del "nuevo orden mundial" institucionalizado en la Organización de las Naciones Unidas, pero ésta nunca desapareció del imaginario estadounidense. ${ }^{51}$ Curiosamente, en esa trayectoria se encontró la Unión Americana con el Reino Unido, y de manera sorprendente con la España de Aznar, que buscó resucitar su historial de imperio colonial que los mismos estadounidenses habían derrotado en la guerra de 1898. En fin, paradojas de la historia.

\section{Referencias bibliográficas}

Autor no especificado, Treaty with Spain (Cession of Porto Rico and the Philippines), en: http://www.bartleby.com/43/46.html

Bender, Meter, “America: The New Roman Empire?", Orbis, vol. 47, núm. 1, invierno, 2003, pp. 145-159.

Chace, James, "Imperial America and the Common Interest", World Policy Journal, primavera, 2002, pp.1-9.

Crenson, Mathew, y Benjamin Ginsberg, Presidential Power: Unchecked and Unbalanced, Nueva York, Norton, 2007, p. 114.

Dorrien, Gary, Imperial Designs: Neoconservatism and the New Pax Americana, Routledge, Nueva York y Londres, 2004, p. 18.

Dougherty, James E., y Robert L. Pfaltzgraff Jr., Teorías en pugna en las Relaciones Internacionales, Grupo Editor Latinoamericano, Buenos Aires, 1993.

Fann, K. T., y Donald C. Hodges (eds.), Readings in U. S. Imperialism, Porter Sargent Publisher, Boston, Massachusetts, 1971, p. 23.

51. Dorrien, Gary, Imperial Designs: Neoconservatism and the New Pax Americana, Routledge, Nueva York y Londres, 2004: 18. 
Ferguson, Niall, Empire: How Britain made the modern world, Penguin Books, Londres, 2003, p. xxi.

Ferling, John, Almost a Miracle, the American Victory in the War of Independence, Oxford University Press, 2007, p. 135.

Freddy, Mark, y Tood Davis, The New Big Book of U.S. Presidents, Courage Books/Running Press, Filadelfia, 2000, p. 55.

Frey, Marc, y Todd Davis, The New Big Book of US Presidents, Courage Books/ Running Press Books Publishers, 2000, p. 33.

Gienapp, Heyrman Davidson, Nation of Nations, a Narrative History of the American Republic, McGraw-Hill Publishing Company, Nueva Cork, 1990, p. 817.

Griffin, Ray, The new Pearl Harbor: Disturbing questions about the Bush administration and 9/11, Olive Branch Press, Massachusetts, 2004.

Gujahr, C. Paul, An American Bible, a History of the Good Book in the United States, 1777-1880, Stanford University Press, Stanford, California, 1999, p. 40.

Henderson, Timothy J., A Glorious Defeat: Mexico and its War with the United States, Hill and Wang, Nueva York, 2007.

Hendrickson, David C., "The Curious Case of American Hegemony: Imperial Aspirations and National Decline", World Policy Journal, vol. xxII, núm. 2, verano, 2005, p. 3.

Irons, Peter, War Powers, How the Imperial Presidency Hijacked the Constitution, Metropolitan Books/Henry Holt and Company, Nueva York, 2005, pp. 88 y 89.

Kirby Martin, James, Randy Roberts, et al., America and its People, vol. 2, Pearson Longman, Nueva York, Boston y San Francisco, 1865.

Kurth, James, "Migration and the Dynamics of Empire" The National Interest, primavera, 2003, pp. 5-16.

LaFeber, Walter, The New Empire: an Interpretation of American Expansion 18601898, Ithaca y Londres, Cornell University Press, 1963, p. vii.

Maryse, Robert, Negotiating NAFTA. Explaining the outcome in culture, textiles, autos, and pharmaceuticals, University of Toronto Press, 2000, p. 26.

Meyer, Lorenzo, y Zoraida Josefina Vázquez, México frente a Estados Unidos (Un ensayo histórico 1776-1988), Fondo de Cultura Económica, México, 1989.

Milkis, Sydeney M., y Michael Nelson, The American Presidency: Origins and Development 1776-2002, cQ Press, Washington, DC, 2003, p. 67. 
Millet, Allan, y Meter Maslowsi, For the Common Defense, a Military History of the United Status of the America, The Free Press, 1994, p. 284.

Montojo Pan, Juan, “ ¿Qué habría ocurrido si España hubiese evitado la guerra con Estados Unidos en 1898?", en Nigel Townson, Historia virtual de España (1870-2004), Taurus, 2004, p. 64.

Nash, Gary B., y Julie Roy Jeffrey (eds.), The American People: creating a Nation and a Society, vol. 2: "Since 1865", Pearson Longman, Nueva York, San Francisco y Boston, 2004.

Nevins, Allan, y Henry Steele, Breve historia de los Estados Unidos, $1^{\text {a }}$ edición en español, Fondo de Cultura Económica, 1994, pp. 359-360.

O'Connor, James, “The Meaning of Imperialism”, en K. T. Fann, y Donald C. Hodges (eds.), Readings in U. S. Imperialism, Porter Sargent Publisher, Boston, Massachusetts, 1971, p. 23.

Ortega y Medina, Juan A., Destino manifiesto: sus razones históricas y su raíz teológica, Alianza Editorial Mexicana/Consejo Nacional para la Cultura y las Artes, México, 1989.

Pérez, Louis A. Jr., The War of 1898: The United States and Cuba in History and Historiography, The University of North Carolina Press, Chapel Hill y Londres, 1998.

Rieff, David, “A New Age of Liberal Imperialism?”, World Policy Journal, verano, 1999, pp. 1-10.

Rosen, Stephen Peter, "An Empire, If You Can Keep It", The National Interest, primavera, 2003, p. 51.

Scott, Len, "International history 1945-1990", en John Baylis, y Steve Smith, The Globalization of World Politics. An Introduction to International Relations, $2^{\mathrm{a}}$ edición, University Press, 2001, p. 76.

Snyder, Jack, "Imperial Temptations", The National Interest, primavera, 2003, pp. 29-40.

Vidal, Gore, Empire: a Novel, Random House, Nueva York, 1987.

_- The Last Empire: Essays 1992-2000, Vintage International, Nueva York, 2001.

Winter, Caroline, The Culture of Classicism: Ancient Greece and Roma in American Intellectual Life 1780-1910, The Johns Hopkins University Press, 2002, p. 9. 